\title{
Extreme value of wind-excited response considering influence of bandwidth
}

\author{
Guoqing Huang $\cdot$ Xinzhong Chen $\cdot$ Mingshui Li $\cdot$ \\ Liuliu Peng
}

Received: 21 March 2013/Revised: 6 May 2013/Accepted: 13 May 2013/Published online: 5 June 2013

(C) The Author(s) 2013. This article is published with open access at Springerlink.com

\begin{abstract}
This paper addresses the peak factors of windexcited responses including alongwind, acrosswind tall building responses and vortex-induced vibration considering the bandwidth parameter. The influence of bandwidth parameter on the peak factor is investigated using advanced upcrossing theory taking the bandwidth influence into account. Results show that Davenport's formula without consideration of bandwidth parameter servers well in general. However, the advanced upcrossing theory leads to a better prediction of the peak factor of wind-induced response of very lightly damped buildings.
\end{abstract}

Keywords Peak factors - Wind-excited responses . Upcrossing theory $\cdot$ Bandwidth

\section{Introduction}

The closed-form formula introduced by Davenport [1] is widely used for estimating the mean extreme value and peak factor of a Gaussian stationary wind-excited structural response. This formula was developed based on the wellknown "Poisson approximation" that the threshold level crossing occurs independently according to a Poisson process. While the Poisson approximation is generally

G. Huang $(\bowtie) \cdot$ M. Li $\cdot$ L. Peng

Research Center for Wind Engineering, School of Civil

Engineering, Southwest Jiaotong University, Chengdu 610031,

Sichuan, China

e-mail: ghuang1001@gmail.com

X. Chen

Wind Science and Engineering Research Center, Department of Civil and Environmental Engineering, Texas Tech University, Lubbock, TX 79409, USA valid for broad band processes, it may fall short for very narrow band processes, and lower threshold levels of practical interest, due to the tendency of crossings to occur in cluster [2, 3]. Cartwright and Longuet-Higgins [4] introduced a bandwidth parameter to account for its effect on the extreme value. Furthermore, Vanmarcke [2] proposed an improved formula with a different bandwidth parameter, which explicitly described the dependence between the crossing events and the time that the process spends above the threshold. Recently, Huang et al. [5] have presented the peak factors considering the bandwidth parameters for the non-Gaussian resultant response.

This study presents a comprehensive study concerning the peak factors of wind-excited responses considering the influence of bandwidth parameter. The responses considered include the displacement and acceleration of tall buildings in both alongwind and acrosswind directions, which are characterized as Gaussian processes, and vortex-induced vibration which is often described by non-Gaussian process due to the existence of nonlinear damping. These responses are calculated in the frequency domain based on the spectral analysis. The bandwidth parameters of these typical windexcited responses are determined from their power spectra. The peak factors are then quantified considering the bandwidth parameters using Vanmarcke's formulation and compared with Davenport's formulation where the bandwidth parameter is neglected. These peak factors are also compared with those directly determined from time history simulations.

\section{Theoretical background}

The cumulative distribution function (CDF) of the extreme value of a stationary Gaussian process $X(t)$ over time duration $T$ can be expressed as follows 
$Q_{\max }(x)=\exp [-v(x) T]$,

where $v(x)$ is the upcrossing rate at level $x$. For a zero-mean stationary Gaussian process, the upcrossing rate can be estimated based on "Poisson assumption"

$v(x)=v_{0} \exp \left(-\frac{x^{2}}{2 \sigma_{x}^{2}}\right)$,

where $v_{0}=\sigma_{\dot{x}} /\left(2 \pi \sigma_{x}\right)$ is the upcrossing rate across zero mean level; $\sigma_{x}$ and $\sigma_{\dot{x}}$ are the standard deviations (STDs) of $X(t)$ and its derivative $\dot{X}(t)$. The Poisson approximation is named based on the fact that if the crossing rate is independent of the past history of the process, then the time lengths between the upcrossings will be independent; this makes the integer-valued process that counts the number of upcrossing in a Poisson process [3].

Davenport [1] derived the following closed-form formula for the mean and STD of the extreme value

$\mu_{x \max }=g \sigma_{x}=(\beta+\gamma / \beta) \sigma_{x}$,

$\sigma_{x \max }=\pi /(\sqrt{6} \beta) \sigma_{x}$,

where $g$ is the peak factor and $\gamma=0.5772$ is the Euler's constant; $\beta=\sqrt{2 \ln \left(v_{0} T\right)}$. The Poisson approximation may result in serious errors for very narrow band processes [3]. In such a situation, the upcrossing of level $x$ at time $t$ is very likely to be associated with another upcrossing approximately one period later, due to the slowly varying amplitude of the process. Such a dependence of the upcrossing time is inconsistent with the Poisson approximation that the time between the two upcrossings is independent [3]. The Poisson approximation is also less accurate for the lower threshold level of practical interest.

To avoid the limitation of Poisson approximation, the Poisson amplitude-crossing model can be used [6]. The Poisson approximation is more suitable for amplitude of the process than for the process itself. In addition, better results for small $x$ values can be made to include the initial conditions. The modified version of amplitude-crossing rate, $\eta(x)$, is estimated as [3]

$\eta(x)=v(x)\left\{1-\exp \left[-\frac{v_{A}(x)}{v(x)}\right]\right\}\left[1-\frac{v(x)}{v_{0 x}}\right]^{-1}$,

where $v_{A}(x)=\sqrt{2 \pi\left(1-\alpha_{1}^{2}\right)} x / \sigma_{X} v(x)$ is the upcrossing rate of amplitude process $A(t)$; and $\alpha_{1}$ is bandwidth parameter and defined as

$\alpha_{1}=m_{1} /\left(m_{0} m_{2}\right)^{1 / 2}$,

$m_{i}=\int_{-\infty}^{\infty}|\omega|^{i} S_{X}(\omega) \mathrm{d} \omega=\int_{0}^{\infty}(2 \pi f)^{i} S_{X}(f) \mathrm{d} f$,

where $m_{i}$ is the $i$ th spectral moment. As $\alpha_{1}$ tends to unity,
$X(t)$ becomes a very narrow band process. Vanmarcke [2] proposed an empirical correction for $v_{A}(x)$ by fitting the simulation data and accordingly, the upcrossing rate is given as

$$
\begin{aligned}
\eta(x)= & v_{0} \exp \left(-\frac{x^{2}}{2 \sigma_{x}^{2}}\right) \times\left\{1-\exp \left(-\sqrt{2 \pi}\left(1-\alpha_{1}^{2}\right)^{0.6} \frac{x}{\sigma_{x}}\right)\right\} \\
& \times\left[1-\exp \left(-\frac{x^{2}}{2 \sigma_{x}^{2}}\right)\right]^{-1} .
\end{aligned}
$$

The improved extreme value distribution is then obtained by replacing $v(x)$ with $\eta(x)$ in Eq. (1). The mean and STD of the extreme value can be estimated numerically.

It is noted that Cartwright and Longuet-Higgins [4] introduced a different bandwidth parameter and derived the peak factor $g$ as

$\varepsilon=\sqrt{1-m_{2}^{2} /\left(m_{0} m_{4}\right)}$,

$g=\sqrt{2 \ln \left[\left(1-\varepsilon^{2}\right)^{1 / 2} v T\right]}+\gamma / \sqrt{2 \ln \left[\left(1-\varepsilon^{2}\right)^{1 / 2} v T\right]}$.

It is obvious that the above formula reduces to Davenport's formulation for $\varepsilon=0$. When $\varepsilon$ approaches to the zero, the process becomes a very narrow band one.

Figure 1 shows the ratio $\eta(x) / v(x)$ as a function of $\alpha_{1}$. Figure 2 shows the improved CDFs, probability density functions (PDFs) from Varmarcke's formula for different $\alpha_{1}$ and those from Poisson assumption under $v_{0} T=10^{3}$. The results show that there are considerable differences in the mean upcrossing rate and corresponding extreme value distribution, especially at low levels of $x$, as $\alpha_{1}$ approaches to unity, i.e., the process becomes narrower. It is also seen that these differences are negligible as $\alpha_{1}$ is $<0.9$. Figure 3 shows the mean, STD, and their ratio of the extreme value evaluated by Davenport's and Vanmarcke's formulas under $v_{0} T=10^{2}, 10^{3}$, and $10^{4}$ for different $\alpha_{1}$. It can be seen that the difference between two formulations is distinctive as the parameter $\alpha_{1}$ increases to unity. For very narrow band processes, the improved Vanmarcke's formula offers a smaller mean and a larger STD of extreme value.

\section{Alongwind building response}

Consider an alongwind response of an isolated tall building with a regular cross section. The PSD of the alongwind generalized force of the fundamental mode is determined based on the Architectural Institute of Japan (AIJ) recommendations [7, 8]. This spectrum involves some assumptions including a liner fundamental mode shape, negligible 


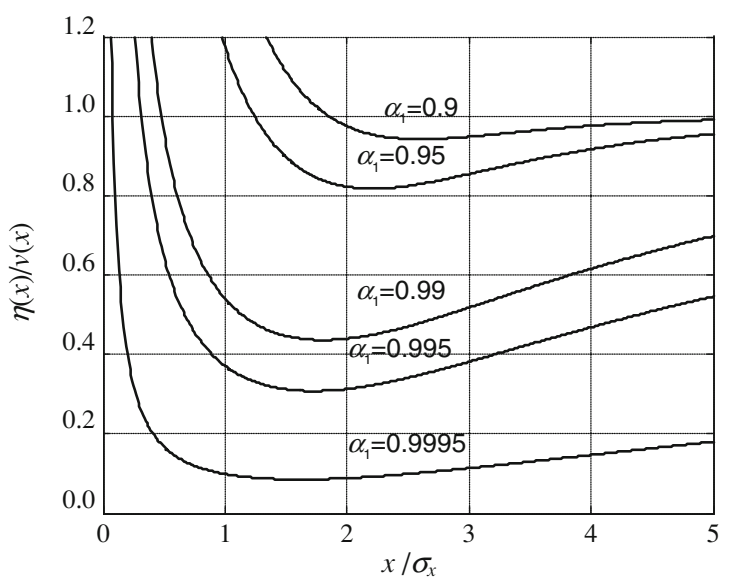

Fig. $1 \eta(x) / v(x)$ as a function of bandwidth parameter $\alpha_{1}$

(a) 1.0

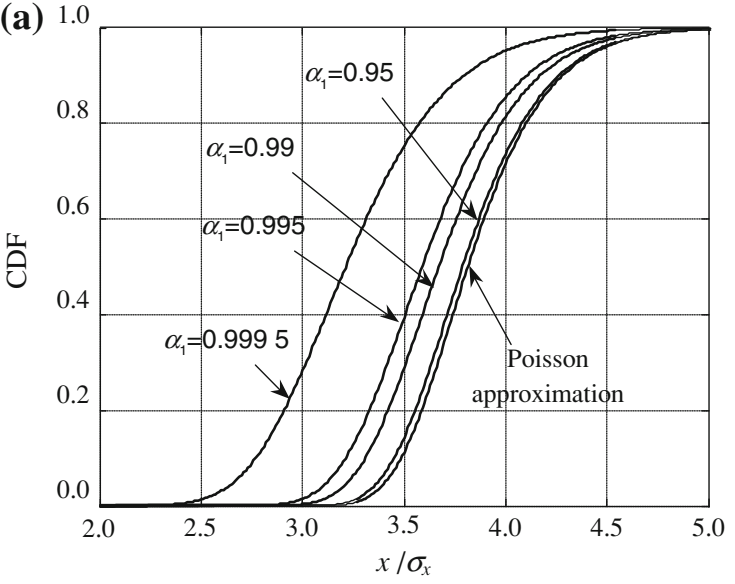

(b)

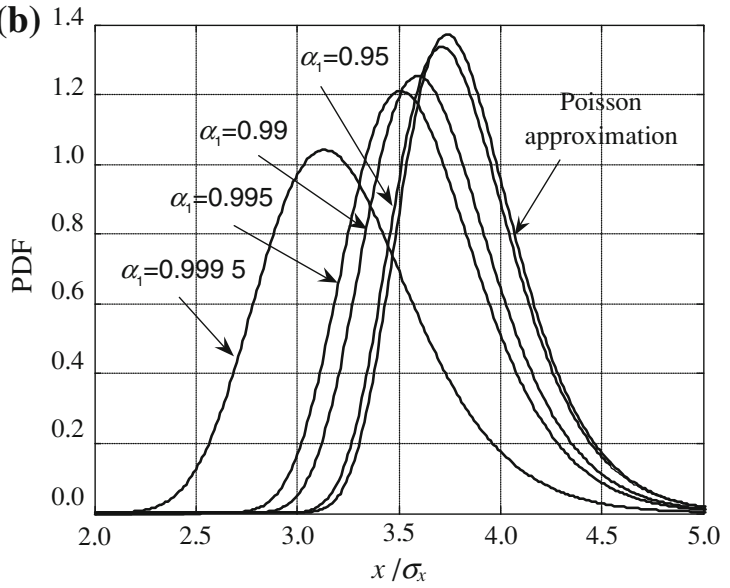

Fig. 2 Probability functions of extreme value. a CDFs $\left(v_{0} T=10^{3}\right)$, b PDFs $\left(v_{0} T=10^{3}\right)$

aerodynamic damping, and a power law profile of the mean wind speed and turbulence intensity. It is expressed as the product of aerodynamic admittance function and the wind fluctuation spectrum as
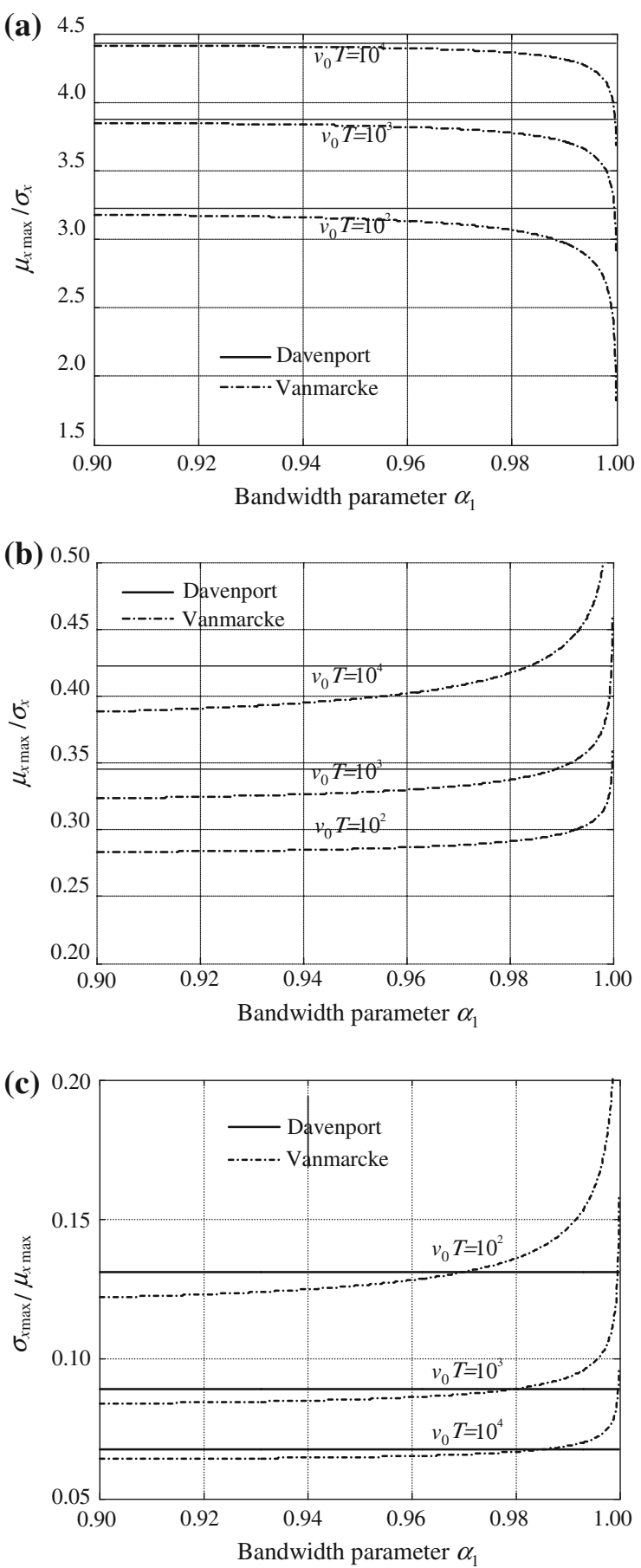

Fig. 3 Influence of bandwidth parameter on extreme value. a Mean of extreme value, b STD of extreme value, and c Coefficient of variation of extreme value

$$
\begin{aligned}
f S_{Q}(f)= & \frac{0.84}{\left(1+2.1 f H / U_{H}\right)\left(1+2.1 f B / U_{H}\right)} \\
& \times \frac{4 f L_{H} / U_{H}}{\left[1+71\left(f L_{H} / U_{H}\right)^{2}\right]^{5 / 6}},
\end{aligned}
$$


where $f$ is the frequency; $L_{H}$ is the turbulence length scale and given as $L_{H}=100 \sqrt{H / 30} ; H$ is building height; $B$ is building width; and $U_{H}$ is the mean wind speed at building top.

Figure 4 shows the PSD of the generalized force $Q(t)$ with $H=200 \mathrm{~m}, B=0.2 H$, and $U_{H}=40 \mathrm{~m} / \mathrm{s}$. Figure 5 portrays the calculated PSDs of the building top displacement and acceleration, i.e., $S_{X}(f)$ and $S_{\ddot{X}}(f)=(2 \pi f)^{4} S_{X}(f)$. The modal damping ratio and natural frequency are taken as $\xi=1 \%$ and $f_{0}=0.23 \mathrm{~Hz}$. As only the fundamental modal response is considered, the PSDs of base shear and based bending moments have the same features as the top displacement.

The building response can be decomposed into the broad band background and narrow band resonant components. It is seen that the PSD of the displacement contains significant background component which is negligible for the acceleration. The PSDs of the background and resonant components of the top displacement are given as

$$
\begin{aligned}
& S_{X_{b}}(f)=S_{Q}(f) /\left[M^{2}\left(2 \pi f_{0}\right)^{4}\right], \\
& S_{X_{r}}(f)=\frac{S_{Q}(f)}{M^{2}(2 \pi)^{4}\left[\left(f_{0}^{2}-f^{2}\right)^{2}+\left(2 \xi f_{0} f\right)^{2}\right]}-S_{X_{b}}(f),
\end{aligned}
$$

where $M$ is the generalized mass. Figure 6 shows the PSDs of the background and resonant components of the top displacement.

Based on the response spectrum, the spectral moments and bandwidth parameter can be calculated. These characteristics of the total response can also be expressed in terms of those of background and resonant components. Their upcrossing rate across zero mean level is defined as $v_{0 s}=\sigma_{\dot{X}_{s}} /\left(2 \pi \sigma_{X_{s}}\right)(s=b, r)$, where $b$ and $r$ denote background and resonant components; $\sigma_{X_{s}}$ is RMS value of the background or resonant component; $\sigma_{\dot{X}_{s}}$ is RMS value of

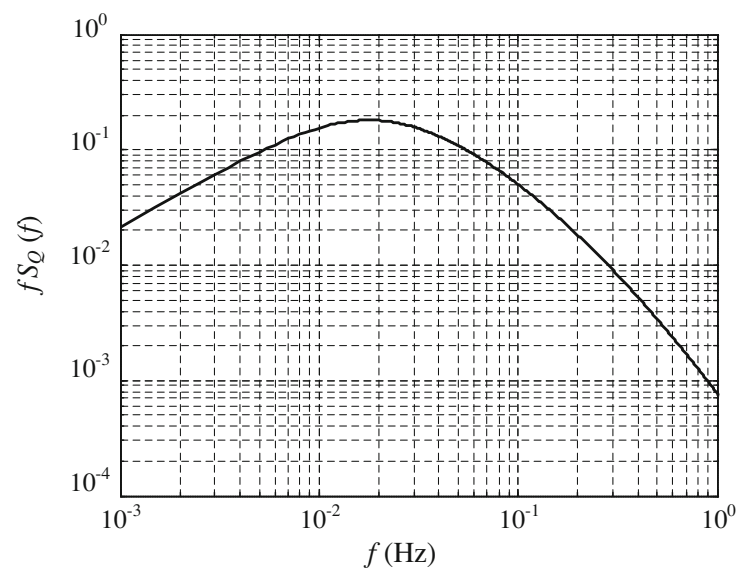

Fig. 4 PSD of the alongwind generalized force
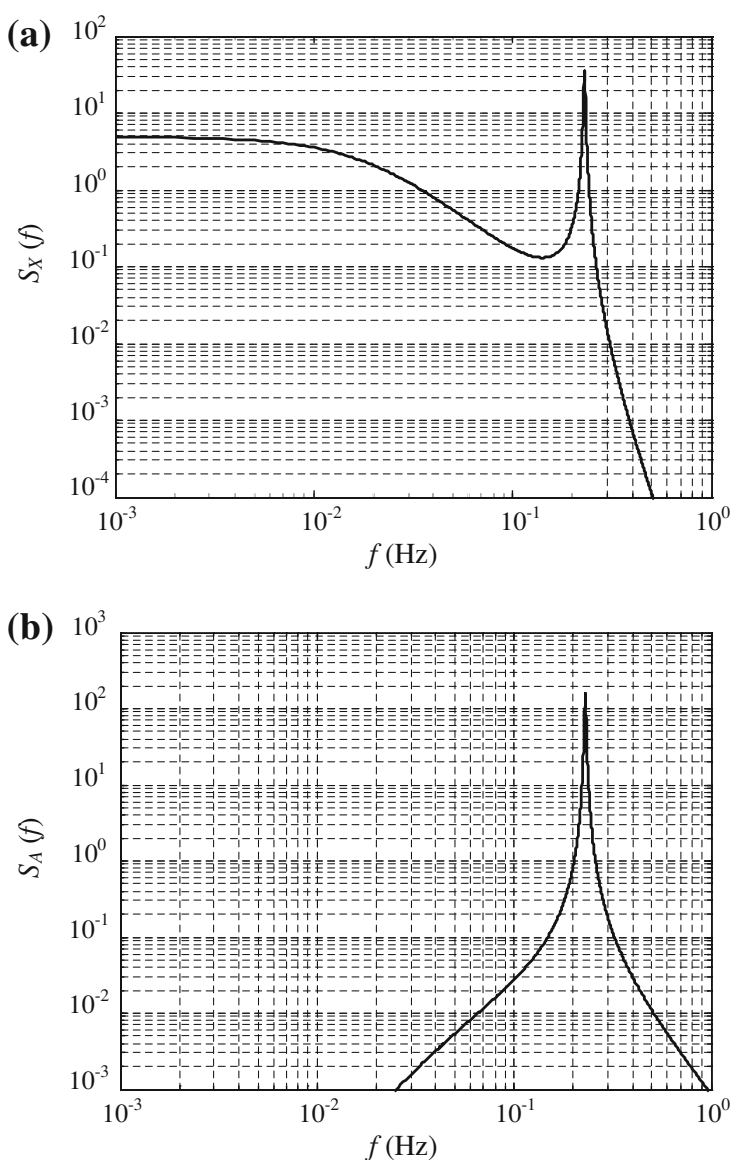

Fig. 5 PSDs of the alongwind top displacement. a Top displacement PSD, b Top acceleration PSD

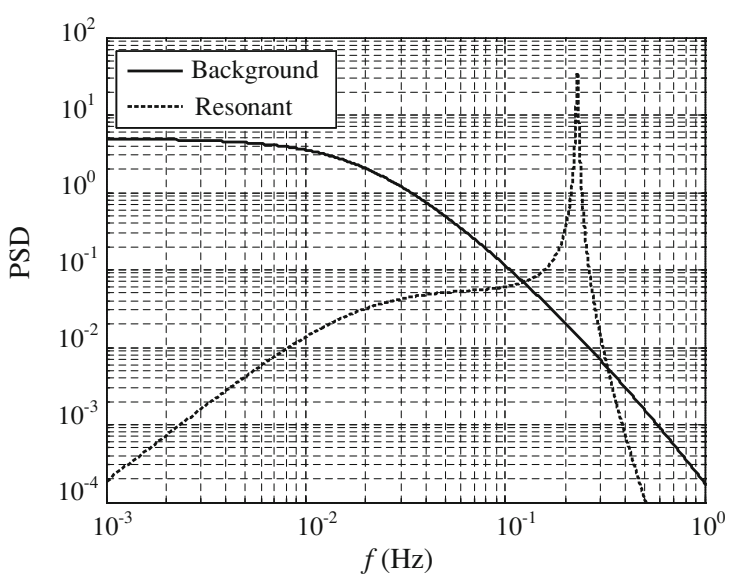

Fig. 6 PSDs of the background and resonant components of top displacement

the derivative of the background or resonant component. Their corresponding bandwidth parameters can be expressed as $\alpha_{1 s}=m_{1 s} /\left(m_{0 s} m_{2 s}\right)^{1 / 2}$, where $m_{i s}=\int_{0}^{\infty}(2 \pi f)^{i} S_{X s}(f) \mathrm{d} f$ $(i=0,1,2 ; s=b, r)$. 
Accordingly, the RMS, upcrossing rate across zero mean level and bandwidth parameter for the total response can be expressed as

$\sigma_{X}=\sqrt{\sigma_{X_{b}}^{2}+\sigma_{X_{r}}^{2}}$

$v_{0}=\sqrt{v_{0 b}^{2}+v_{0 r}^{2}\left(\sigma_{X_{r}} / \sigma_{X_{b}}\right)^{2}} / \sqrt{1+\left(\sigma_{X_{r}} / \sigma_{X_{b}}\right)^{2}}$

$\alpha_{1}=\left[v_{0 b} \alpha_{1 b}+v_{0 r} \alpha_{1 r}\left(\sigma_{X_{r}} / \sigma_{X_{b}}\right)^{2}\right] /\left[v_{0}\left(1+\left(\sigma_{X_{r}} / \sigma_{X_{b}}\right)^{2}\right)\right]$,

where Eqs. (15) and (16) can be derived from the definitions.

Figure 7 shows the ratio of RMS resonant displacement to RMS background displacement as a function of damping ratio. It is seen that the portion of resonant component increases with the decrease in the damping ratio.

Figure 8 shows the bandwidth parameter $\alpha_{1}$ as a function of damping ratio $\xi$ for the displacement and acceleration. Figure 9 shows the relationship between the upcrossing rate across zero mean and damping ratio. The bandwidth parameter $\alpha_{1}$ and uprcossing rate across zero mean for the background response are 0.627 and 0.043 respectively which are independent of the damping ratio. It can be seen that the displacement can be regarded as a broad band except very low-damping ratios where the resonant component becomes dominant. As expected, the acceleration and resonant displacement can be considered as narrow band for the damping ratio range of $1 \%-3 \%$. It is noted that the upcrossing rate of the acceleration and resonant displacement across zero mean can be approximated as the structural natural frequency, i.e., $v_{0 r} \approx f_{0}$.

Figure 10 shows the comparison of the peak factors of alongwind response computed from Davenport's and Vanmarcke's formulations, i.e., without and with consideration of bandwidth parameter. The results illustrates that

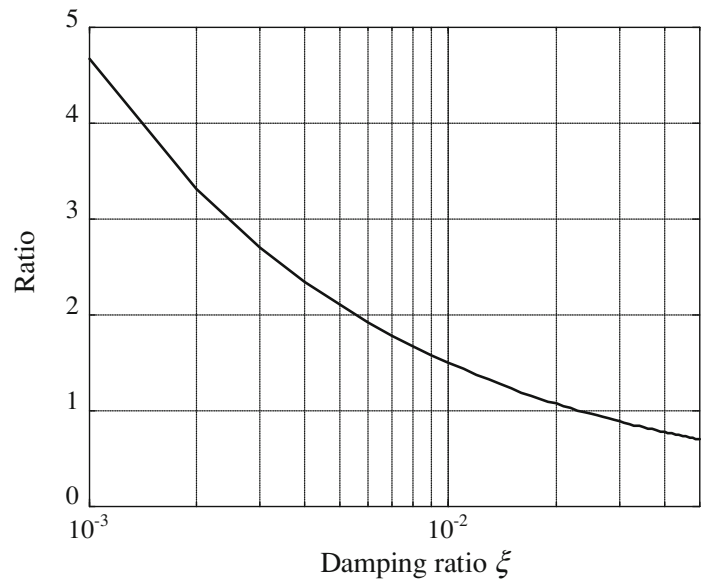

Fig. 7 Ratio of $\sigma_{X_{r}} / \sigma_{X_{b}}$

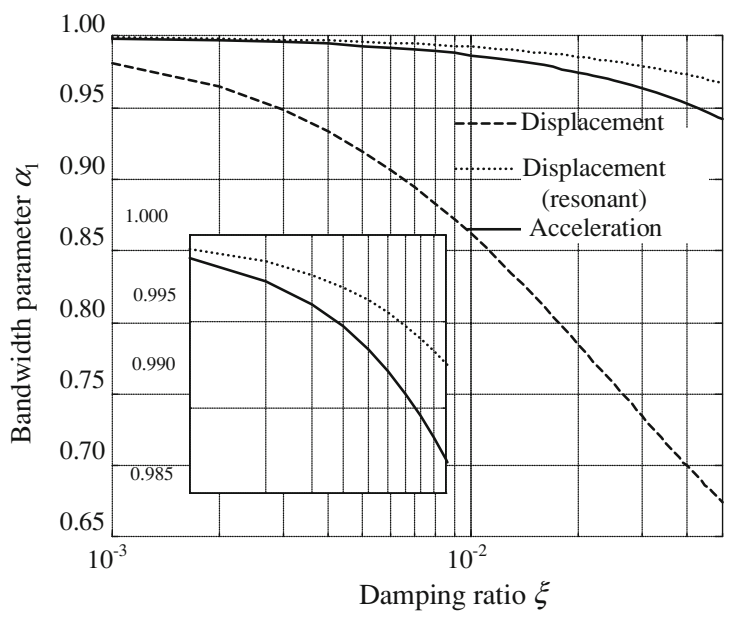

Fig. 8 Bandwidth parameter as a function of damping ratio

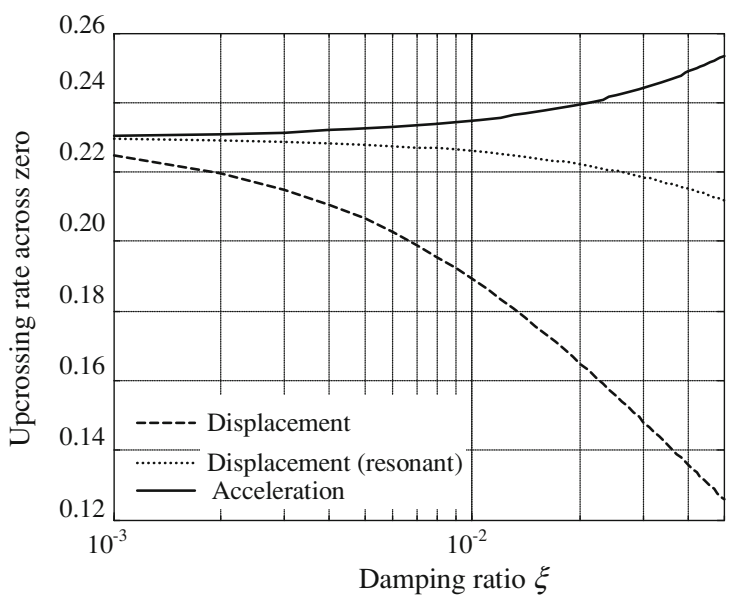

Fig. 9 Relationship between upcrossing rate across zero and damping ratio

the bandwidth parameter has a negligible influence on the displacement. For the acceleration, the consideration of bandwidth parameter results in $4 \%$ difference at $\xi=1 \%$, and $12 \%$ difference at $\xi=0.1 \%$.

In order to validate these formulations, Monte Carlo simulation of building response is also performed. A total of 100 samples of the time histories of the generalized force are generated using spectral representation method [9]. The building response is then calculated using step-bystep Newmark's method. The peak factors of top displacement and acceleration are qualified from the response time histories as shown in Fig. 10. It is seen that the peak factors obtained from the time domain simulation is slightly lower than that from Vanmarcke's formulation. Such a slight difference has also been reported in literature [3].

For the broad band background displacement with a bandwidth parameter of 0.627 , both Davenport's and 

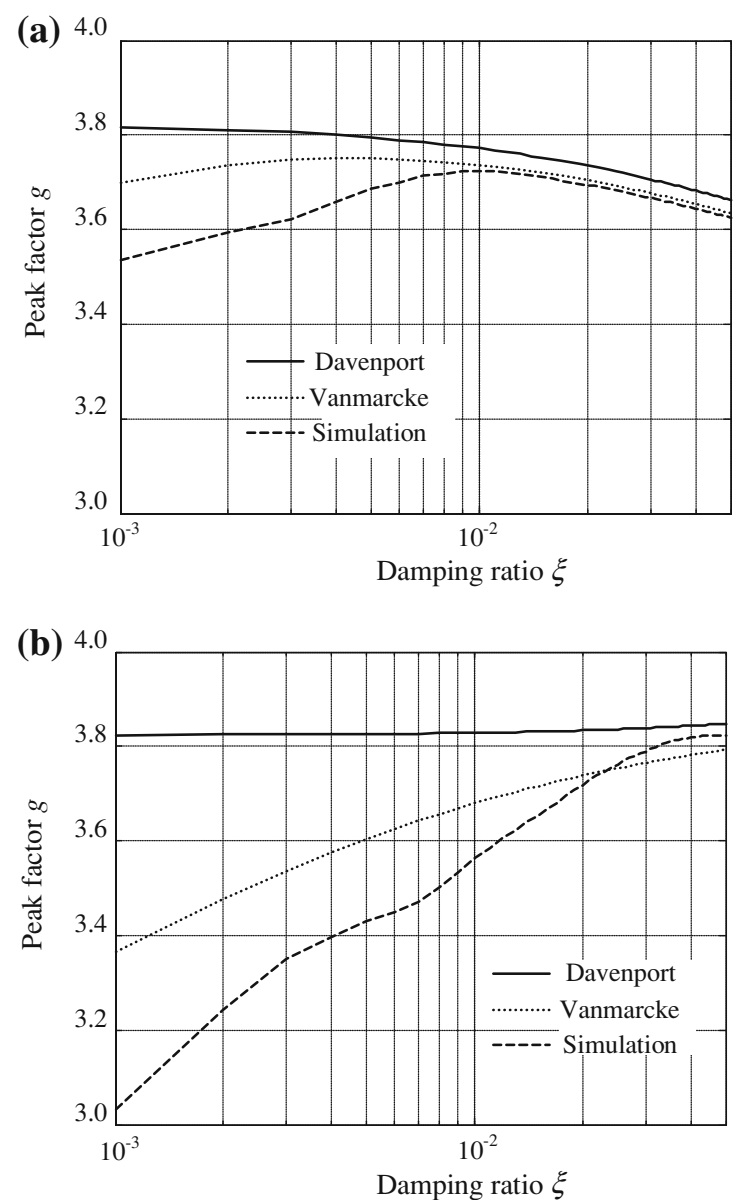

Fig. 10 Influence of bandwidth parameter on the peak factor of alongwind response. a Top displacement, b Top acceleration

Vanmarcke's formulas offer an identical peak factor of 3.22. However, they lead to a slightly different peak factor for the resonant displacement as shown in Fig. 11. As expected, the peak factor of the resonant displacement shows the similar characteristics as that of the acceleration.

In current practice, the mean extreme response is often estimated by directly combining the mean extreme values of background and resonant components as

$\mu_{x \max }=\sqrt{g_{b}^{2} \sigma_{X_{b}}^{2}+g_{r}^{2} \sigma_{X_{r}}^{2}}$,

where $g_{b}$ and $g_{r}$ are the peak factors of background and resonant components.

As compared to the following equation

$\mu_{x \max }=g_{X} \sqrt{\sigma_{X_{b}}^{2}+\sigma_{X_{r}}^{2}}$,

where $g_{X}$ is the peak factor of total response, Eq. (17) is equivalent to introduce a peak factor for total response as

$g_{X}^{\prime}=\sqrt{g_{b}^{2} \sigma_{X_{b}}^{2} / \sigma_{X}^{2}+g_{r}^{2} \sigma_{X_{r}}^{2} / \sigma_{X}^{2}}$.

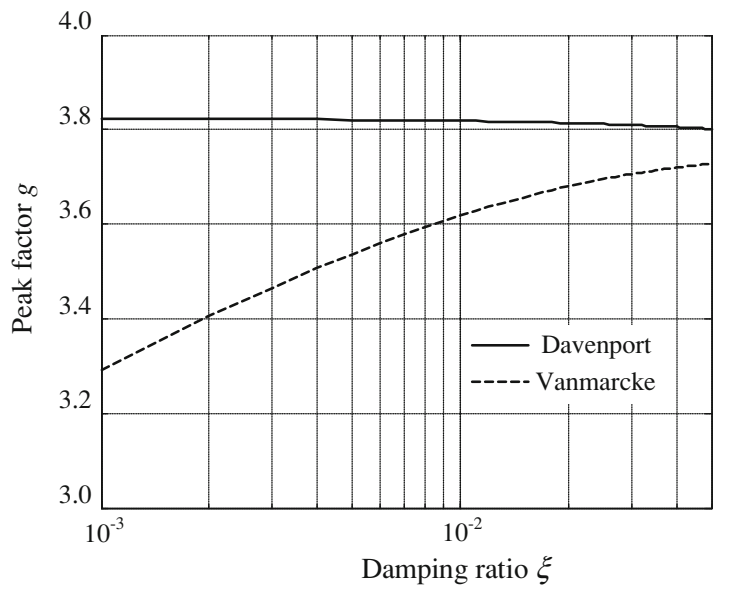

Fig. 11 Influence of bandwidth parameter on the peak factor of resonant top displacement

Figure 12 shows the ratio $g_{X}^{\prime} / g_{X}$ for the total displacement. It can be seen that when Eq. (17) is applied, Davenport's formula leads to a better estimation for the peak factor of total response. The reason is attributed to following coincidence: on one hand, approximate combination of Eq. (17) leads to a lower extreme value of total response when both background and resonant extreme vales are computed using Vanmarcke's formula; on the other hand, the extreme value of resonant component is overestimated by Davenport's formula.

As shown in Eq. (10), Cartwright and Longuet-Higgins [4] introduced a different bandwidth parameter $\varepsilon$ in estimating the peak factor. Figure 13 shows the bandwidth parameter $\varepsilon$ as a function of damping ratio for the top displacement and acceleration. Figure 14 compares the influence of this parameter on the responses. It can be seen that Cartwright and Longuet-Higgins' formulas gives an

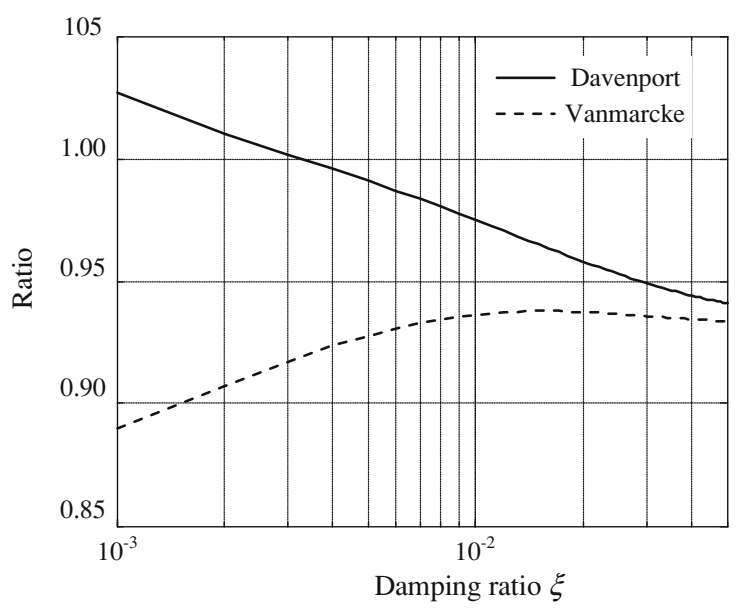

Fig. 12 Ratio of peak factors $g_{X}^{\prime} / g_{X}$ 


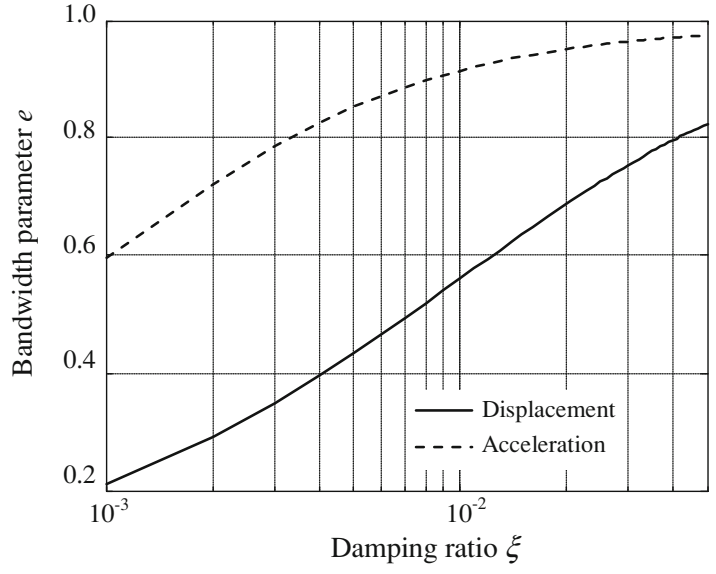

Fig. 13 Relationship between parameter $\varepsilon$ and ratio $\xi$


Fig. 14 Influence of bandwidth parameter $\varepsilon$ on the peak factor of alongwind response. a Top displacement, b Top acceleration

opposite trend of the peak factor with the influence of the bandwidth parameter. Hence, the usage of the parameter $\varepsilon$ is not effective on the estimation of the peak factor and extreme response.

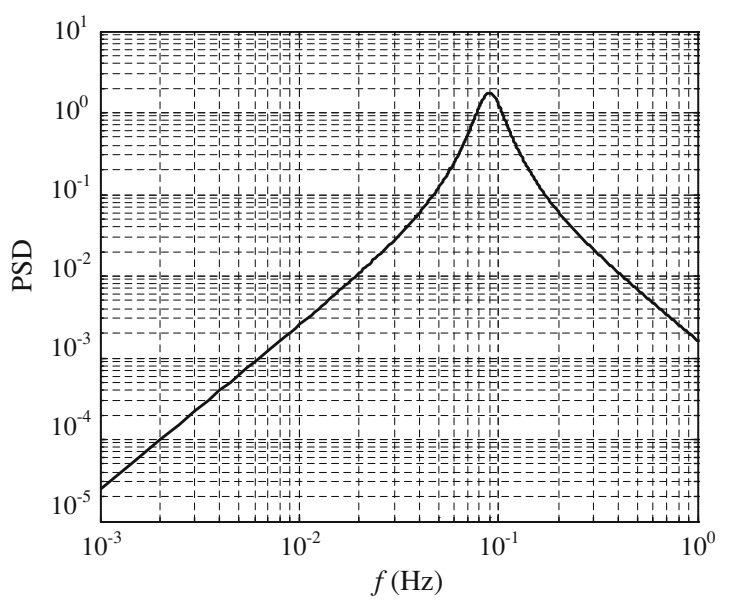

Fig. 15 PSD of the acrosswind generalized force

\section{Acrosswind building response}

The PSD of the acrosswind generalized force $Q(t)$ suggested by AIJ recommendations (AIJ 1996) [7] is also used for estimating the acrosswind response and discussing the bandwidth parameter on its peak factor and extreme value.

$$
\begin{array}{r}
S_{Q}(f)=\sum_{j=1}^{N} \frac{4 \kappa_{j}\left(1+0.6 \beta_{j}\right) \beta_{j} \times\left(f / f_{s j}\right)^{2}}{\pi \times\left\{\left[1-\left(f / f_{s j}\right)^{2}\right]^{2}+4 \beta_{j}^{2}\left(f / f_{s j}\right)^{2}\right\}}, \\
N=\left\{\begin{array}{cc}
1, D / B<3 \\
2, D / B>3
\end{array}, \quad\left\{\begin{array}{l}
\kappa \approx 0.85 \\
\kappa \approx 0.02
\end{array},\right.\right.
\end{array}
$$

where $\beta_{j}$ is related to the band width; $f_{s j}$ represents the peak frequencies, i.e., vortex-shedding frequencies of the spectrum; and $D$ is the building depth. They can be determined by the following empirical formulas as functions of the side ratio $D / B$ ( $B$ is the building width)

$$
\begin{aligned}
f_{s 1} & =\frac{0.12}{\left[1+0.38(D / B)^{2}\right]^{0.89}} \frac{U_{H}}{B}, \\
f_{s 2} & =\frac{0.56}{(D / B)^{0.85}} \frac{U_{H}}{B} \\
\beta_{1} & =\frac{(D / B)^{4}}{1.2(D / B)^{4}-1.7(D / B)^{2}+21}+\frac{0.12}{D / B}, \\
\beta_{2} & =0.28(D / B)^{-0.34} .
\end{aligned}
$$

Figure 15 shows the PSD of acrosswind generalized force with $H=200 \mathrm{~m}, B=0.2 H, D / B=1$, and $U_{H}=40 \mathrm{~m} / \mathrm{s}$. Figure 16 shows the PSDs of the building top displacement and acceleration with damping ratio $\xi=1 \%$.

Figure 17 shows the bandwidth parameter $\alpha_{1}$ for the displacement and the acceleration as a function of damping ratio. Figure 18 shows the relationship between the upcrossing rate across zero and damping ratio. It can be seen 

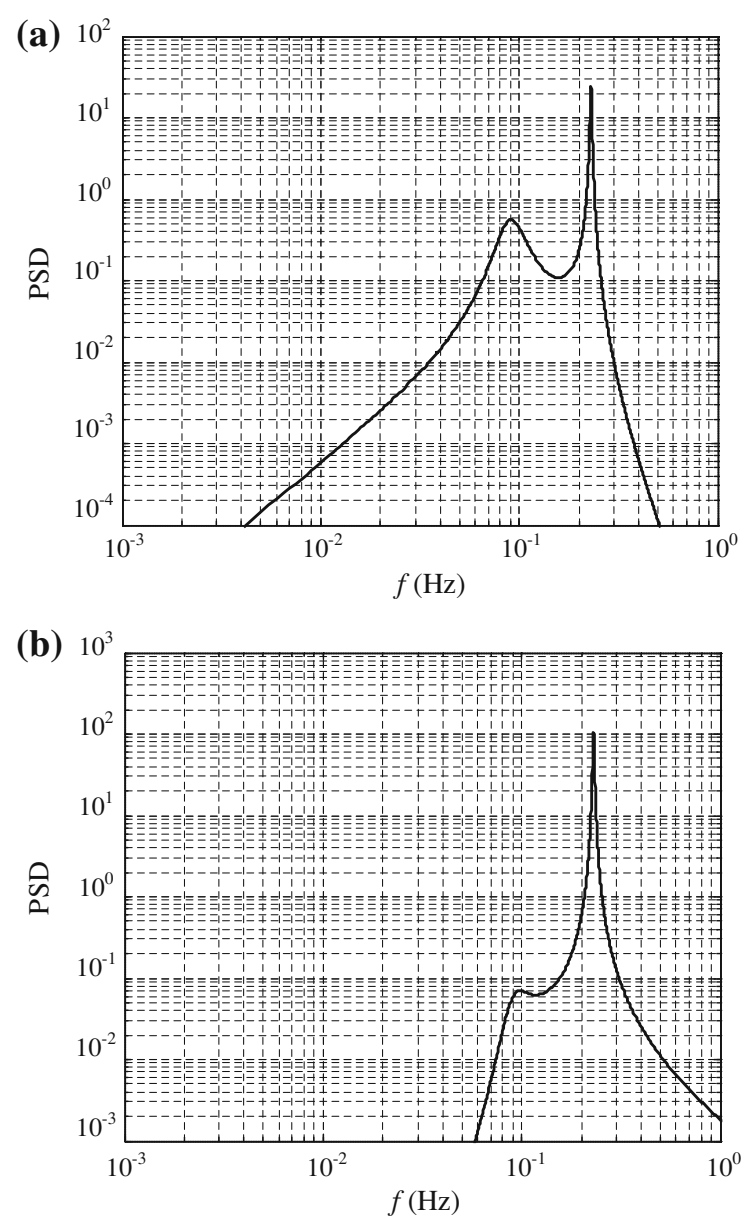

Fig. 16 PSDs of the acrosswind displacement and acceleration $(\xi=1 \%)$. a Top displacement, b Top acceleration

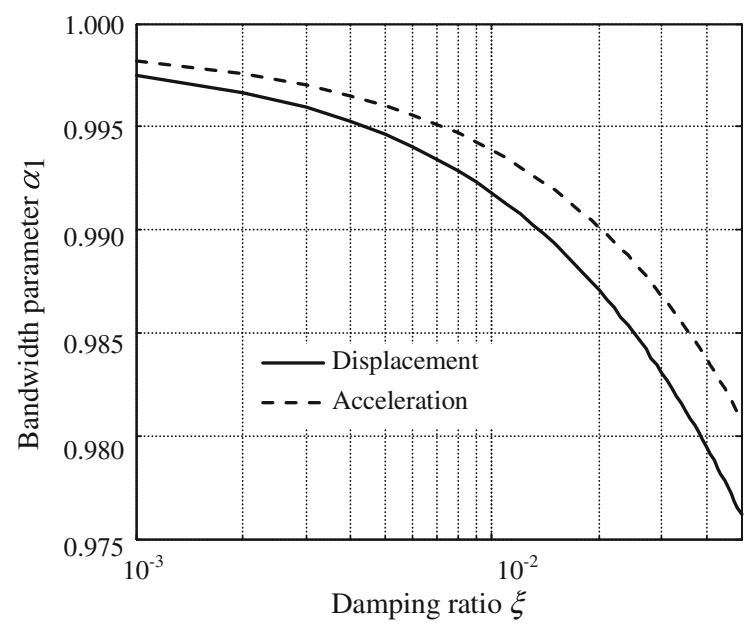

Fig. 17 Relationship between bandwidth parameter and damping ratio

that the displacement and acceleration can be considered as narrow band processes, and the upcrossing rate across zero level can be approximated by the natural frequency.

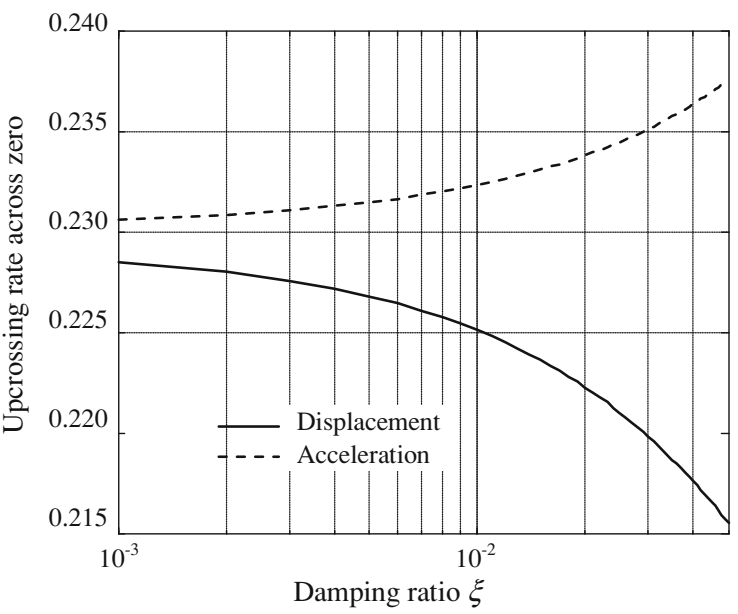

Fig. 18 Relationship between upcrossing rate and damping ratio

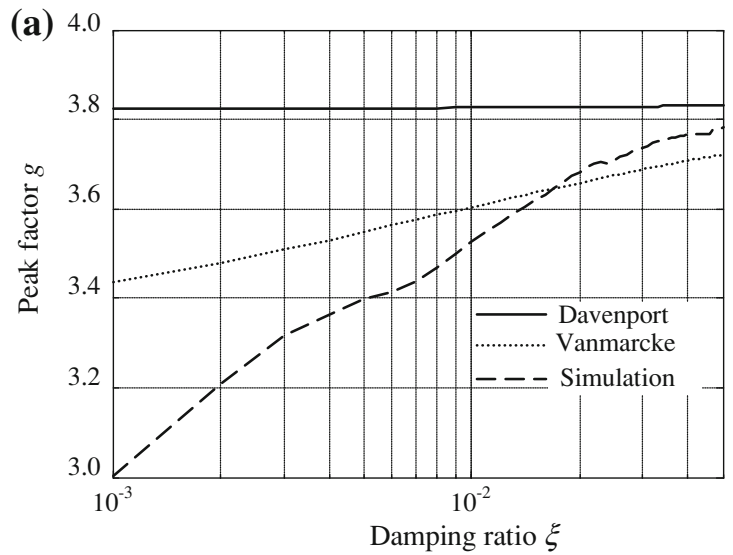

(b) 4.0

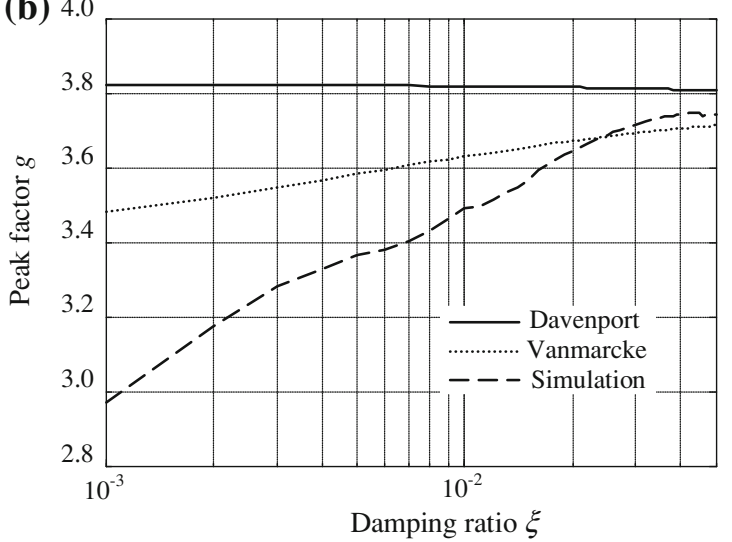

Fig. 19 Influence of bandwidth parameter on the peak factor of acrosswind response. a Top displacement, b Top acceleration

Figure 19 shows the influence of bandwidth parameter on the peak factor of acrosswind response. The results illustrate that the consideration of bandwidth parameter results in $6 \%$ and $11 \%$ differences at $\xi=1 \%$ and $0.1 \%$ for displacement, respectively. The differences are $5 \%$ and $9 \%$ at $\xi=1 \%$ and $0.1 \%$ for acceleration, respectively. It 
is seen that Vanmarcke's formula will provide a better prediction of the peak factor of wind-induced response of very lightly damped buildings.

\section{Vortex-induced response}

Consider a vortex-induced vibration of a spring-supported circular cylinder. A considerable variety of empirical analytical models have been developed to describe the vortex-induced vibration of cylinders [10]. In this study, the equation of vibration of a cylinder with a unit length is given as [11]

$$
\begin{aligned}
m\left(\ddot{y}+2 \xi \omega_{1} \dot{y}+\omega_{1}^{2} y\right)= & \frac{1}{2} \rho U^{2} D\left[Y_{1}(K) \times\left(1-\delta \frac{y^{2}}{D^{2}}\right) \frac{\dot{y}}{U}\right. \\
& \left.+Y_{2}(K) \frac{y}{D}+C_{L}(K) \sin (\omega t+\phi)\right],
\end{aligned}
$$

where $m$ is the mass per unit length of cylinder; $\xi$ and $\omega_{1}$ are damping ratio and natural circular frequency respectively; $\rho$ is air density; $D$ is diameter of cylinder; $K=\omega D / U$ is reduced frequency; $Y_{1}, \delta, Y_{2}$, and $C_{L}$ are parameters and functions of $K$ and need to be determined through the observations.

This model allows the consideration of nonlinear windexcited "negative damping". At lock-in, $\omega \approx \omega_{1}, Y_{2} \approx 0$, and $C_{L} \approx 0$, since the last two terms are found to be small compared to the first term reflecting the aerodynamic damping effects. $Y_{1}$ and $\delta$ are independent parameters; the former determines the initial negative damping, and the latter dominates the time to achieve the steady-state vibration. The steady-state amplitude of vortex-induced vibration, $y_{0}$, is given as [11]

$\frac{y_{0}}{D}=2\left[\frac{Y_{1}-8 \pi S_{c r} S_{t}}{\delta Y_{1}}\right]^{1 / 2}$,

where $S_{t}$ is Strouhal number; and $S_{c r}=\xi m /\left(\rho D^{2}\right)$ is Scruton number.

In this study, the Strouhal and Scruton numbers are taken as 0.2 and 1.5 , respectively. $Y_{1}$ and $\delta$ are assumed to be 26.3 and 2,928.4, respectively. Accordingly, $Y_{0} / D=0.0312$.

Using the normalized displacement $y^{\prime}=y / D$, Eq. (23) can be can be rewritten as

$$
\ddot{y}^{\prime}+2 \omega_{1}\left[\xi-\frac{U}{4 D \omega_{1}} \frac{\xi}{S_{c r}} Y_{1}\left(1-\delta y^{\prime 2}\right)\right] \dot{y}^{\prime}+\omega_{1}^{2} y^{\prime}=0,
$$

The time history of vortex-induced vibration can be simulated by solving Eq. (25) through Newmark's step-bystep method. Figure 20 shows an example with $\xi=1 \%$ and $\omega_{1}=\pi \mathrm{rad} / \mathrm{s}$. Using the time history data when vortexinduced vibration reaches its steady state, the peak factor is

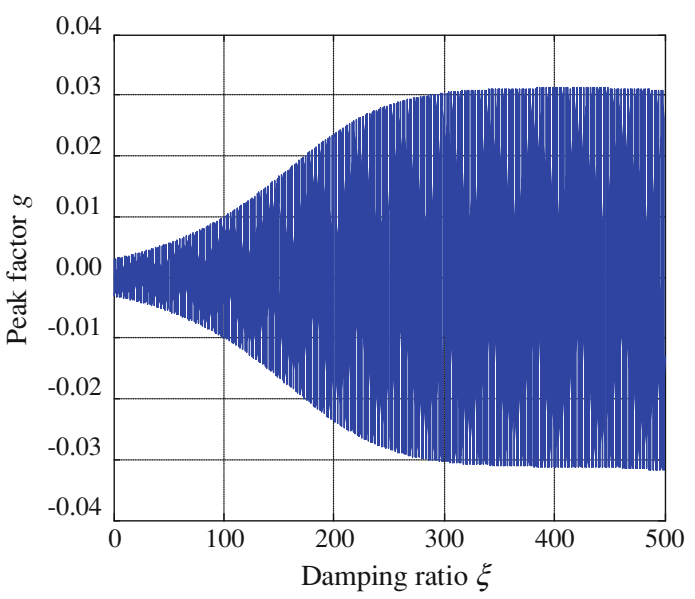

Fig. 20 A sample of vortex-induced vibration

determined to be around 1.42 , which is close to that of a sinusoidal response, $\sqrt{2}$. In this situation, the bandwidth parameter $\alpha_{1}$ should be very close to unity. It should be noted that at the wind speed around lock-in region, vortexshedding frequency is close to the natural frequency of cylinder. The vibration will show the beating phenomena. The peak factor of vortex-induced vibration will be in the range of 1.42 to around 3.5. The advanced theory that takes into account the bandwidth parameter is able to interpret the trend of extreme value and peak factor in this response.

\section{Conclusion}

In wind engineering community, Davenport's closed-form formula was extensively applied in extreme value estimation of a Gaussian stationary response. Due to the "Poisson approximation" involving this formulation, it may fall short for very narrow band processes and lower threshold levels of practical interest. On the other hand, Vanmarcke's formula accounted for the effects of bandwidth and led to an improved estimation of the extreme value of very narrow band processes.

The wind-induced building response was generally separated into background and resonant components. The background response was regarded as a broad band process, while the resonant response was a narrow band process. The bandwidth parameter of the total response was affected by the ratio of resonant and background components. The alongwind displacement usually contained considerable background component. On the other hand, the alongwind acceleration, and acrosswind displacement and acceleration were dominant by the resonant component. The bandwidth parameter was strongly affected by the building damping ratio. Vortex-induced vibration at steady-state stage had a bandwidth parameter which was 
very close to unity because its vibration was almost a sinusoidal wave.

The estimation of the extreme value and peak factor using Davenport's formulation without considering the bandwidth parameter served well for both alongwind and acrosswind responses. It may lead to an around $6 \%$ overestimation for the acrosswind response of buildings with very lightly damping ratios, say, $1 \%$. The time domain simulations validated those results.

However, Vanmarcke's formulation with the consideration of bandwidth parameter and the lower level of crossing was more accurate in describing the probability distribution of the extreme value of very narrow band processes. One such an example was vortex-induced vibration. At the steady-state stage, the peak factor was around 1.42. Around the lock-in range, the peak factor was between 1.42 and around 3.5. That was much lower than that computed by Davenport's formula. The advanced theory using Vanmarcke's formula could explain the behavior of very narrow band response processes.

In engineering applications, the peak value of windexcited response was often estimated by directly combining the peak values of its background and resonant components using square-root-of-sum-of-squares (SRSS) rule. Such an approximate combination generally led to a smaller combined peak response when both extremes of the background and resonant components were accurately determined following Vanmarcke's formula. On the other hand, this approximate combination led to a better result when the peak factors were given by Davenport's formula. However, when the resonant response was dominant, the combination of SRSS rule and Davenport's formula may fall short in predicting the extreme response of very lightly damped structures.
Acknowledgments The partial support by the National Science Foundation of China (Grant 51278433) is greatly acknowledged.

Open Access This article is distributed under the terms of the Creative Commons Attribution License which permits any use, distribution, and reproduction in any medium, provided the original author(s) and the source are credited.

\section{References}

1. Davenport AG (1964) Note on the distribution of the largest value of a random function with application to gust loading. Proceedings of the Institution of Civil Engineers. pp187-196

2. Vanmarcke EH (1975) On the distribution of the first-passage time for normal stationary random processes. J Appl Mech $14: 217-243$

3. Lutes LD, Sarkani S (2004) Random Vibration: Analysis of Structural and Mechanical Systems. Elsevier, NewYork

4. Cartwright DE, Longuet-Higgins MS (1956) The statistical distribution of the maxima of a random function. Proc Royal Soc Lond A 237:212-232

5. Huang MF, Chan CM, Lou WJ, Kwok KCS (2012) Statistical extremes and peak factors in wind-induced vibration of tall buildings. J Zhejiang University Sci A 13(1):18-32

6. Cramér H, Leadbetter MR (1967) Stationary and Related Stochastic Processes. Wiley, NewYork

7. Architectural Institute of Japan (1996) AIJ Recommendations for Loads on Buildings, Japan

8. Kijewski T, Kareem A (1998) Dynamic wind effects: a comparative study of provisions in codes and standards with wind tunnel data. Wind Struct 1:77-109

9. Shinozuka M, Jan CM (1972) Digiatal simulation of random processes and its applications. J Sound Vib 25(1):111-128

10. Gabbai RD, Benaroya $H$ (2005) An overview of modeling and experiments of vortex-induced vibration of circular cylinders. J Sound Vib 282:575-616

11. Simiu E, Scanlan RH (1996) Wind Effects on Structures: Fundamentals and Applications to Design, 3rd edn. Wiley, NewYork 